\title{
Metabolische Nebenwirkungen Antipsychotika bei
Kindern: Vorsicht Diabetes!
}

Jugendliche und junge Erwachsene entwickeln unter Antipsychotika gehäuft einen Typ-2-Diabetes. Das liegt nur zum kleinen Teil an der psychischen Störung - ein erheblicher Part des Risikos lässt sich auf die Medikation zurückführen.

Die Lebenserwartung ist bei Schizophreniepatienten um 20-30 Jahre verkürzt. Neben dem Tabakkonsum und den Folgen liegt dies vor allem am erhöhten Risiko für kardiometabolische Erkrankungen, wie eine vor Kurzem veröffentlichte Analyse nahelegt. Gründe für das erhöhte Risiko sind ungesunder Lebensstil und genetische Faktoren, aber auch die Therapie mit Antipsychotika trägt dazu bei. So sind Gewichtszunahme, reduzierte Glukosetoleranz, Insulinresistenz und Dyslipidämie bekannte Begleiterscheinungen einer Behandlung mit modernen Neuroleptika bei Erwachsenen. Es gibt Hinweise, dass solche Effekte bei Jugendlichen noch verstärkt auftreten. „Kardiometabolische Nebenwirkungen scheinen schneller und in größerem Ausmaß aufzutreten als bei Erwachsenen. Sie führen zu einer relevanten Gewichtszunahme bei einem signifikanten Anteil der Jugendlichen“, schreiben Psychiater um Dr. Britta Galling vom Zucker Hillside Hospital in Glen Oaks.

Da Antipsychotika nicht nur im Schizophrenie-Spektrum, sondern zunehmend auch bei anderen Erkrankungen wie bipolaren Störungen verordnet werden, solle verstärkt an die möglichen Konsequenzen gedacht werden.

\section{Studien analysiert}

Mit welchen Konsequenzen Ärzte rechnen müssen, haben die Psychiater um Galling durch eine Metaanalyse evaluiert. Sie berücksichtigten dabei nur solche Studien, in denen bei mindestens 20 Patienten im Alter von weniger als 24 Jahren ein

Bei ihm kann der Einsatz von Antipsychotika ernste Folgen haben.
Typ-2-Diabetes während einer Antipsychotikabehandlung diagnostiziert worden war. Insgesamt stießen sie auf 13 Untersuchungen mit über 1,8 Millionen Teilnehmern, darunter waren über 185.000 Minderjährige und junge Erwachsene mit Behandlung durch Antipsychotika.

Im Schnitt war die Zielgruppe der jungen Erwachsenen und Kindern 14 Jahre alt, knapp 60\% waren männlich. Die meisten hatten die Antipsychotika aufgrund von Verhaltensstörungen, ADHS oder Depressionen bekommen, nur 16\% aufgrund einer bipolaren Störung und rund 6\% aufgrund einer Psychose. $94 \%$ erhielten atypische Neuroleptika (42\% Risperidon, 27\% Quetiapin, 17\% Aripiprazol, 10\% Olanzapin).

Über alle Studien gemittelt erkrankten während der durchschnittlichen Beobachtungsdauer von 2,3 Jahren 5,7 von 1.000 jungen Patienten mit Antipsycho- 